\title{
ASYMPTOTIC SOLUTIONS OF TOROIDAL SHELL PROBLEMS*
}

\author{
BY \\ R. A. CLARK \\ Case Institute of Technology and Massachusetts Institute of Technology
}

1. Introduction. A few years ago a method of asymptotic integration was developed by $\mathrm{E}$. Reissner and the author for a non-homogeneous differential equation involved in certain problems of the theory of thin elastic toroidal shells. Using this method solutions were found for the problem of bending a curved tube (considered as a sector of a shell of revolution) [4] and for the mathematically similar problem of a toroidal expansion joint subject to an axial force [2]. The main purpose of this paper is to indicate how the method used before can be refined and how the approximate solutions previously obtained for these problems can be generalized and extended.

It is shown, in particular, that the previous solutions are the leading terms of asymptotic expansions in inverse powers of a large parameter. Higher-order terms or corrections of two different types are obtained for toroidal shells of circular cross section and uniform thickness. Some of the results are found by specializing solutions derived first for toroidal shells of arbitrary cross section and nossibly varying thickness, assuming on $; 11, n t$ the shell has a plane of symmetry perpendicular to the axis of revolution.

2. Basic equations for shells of revolution. The problem of determining rotationally-symmetric stress distributions in thin elastic shells of revolution may be reduced to that of solving two coupled second-order differential equations. Using the formulation and essentially the notation of E. Reissner [9] the differential equations for small deformations may be written in the form

$$
\begin{aligned}
& X^{\prime \prime}-\theta_{1} X+\mu \Phi Y=F, \\
& Y^{\prime \prime}-\Theta_{2} Y-\mu \Phi X=G .
\end{aligned}
$$

Here primes denote differentiation with respect to an independent variable $\xi$ which is the parameter in a representation of a meridian of the middle shell surface by equations of the form

$$
r=r(\xi), \quad z=z(\xi),
$$

where $r, \theta, z$ are the cylindrical coordinates of a point on the middle surface. Representation (2) is assumed to be such that $r$ and $z$ have continuous derivatives up to at least third order and also such that the quantity

$$
\alpha=\left[\left(r^{\prime}\right)^{2}+\left(z^{\prime}\right)^{2}\right]^{1 / 2}
$$

*Received February 15, 1957. Sponsored in part by the Office of Ordnance Research, U. S. Army, under contract with Case Institu'te of Technology and in part by the Office of Naval Research, U. S. Navy, under contract with Massachusetts Institute of Technology. 
never vanishes. The coefficients and loading terms in Eqs. (1) are given by

$$
\begin{aligned}
\theta_{1}=\left(\frac{r^{\prime}}{r}\right)^{2}-\nu \frac{\left(r^{\prime} D / \alpha\right)^{\prime}}{(r D / \alpha)}+\frac{1}{4}\left[\frac{(r D / \alpha)^{\prime}}{(r D / \alpha)}\right]^{2}+\frac{1}{2}\left[\frac{\left(r D^{\prime} \alpha\right)^{\prime}}{(r D / \alpha)}\right]^{\prime}, \\
\Theta_{2}=\left(\frac{r^{\prime}}{r}\right)^{2}+\nu \frac{\left(r^{\prime} / \alpha C\right)^{\prime}}{(r / \alpha C)}+\frac{1}{4}\left[\frac{(r / \alpha C)^{\prime}}{(r / \alpha C)}\right]^{2}+\frac{1}{2}\left[\frac{(r / \alpha C)^{\prime}}{(r / \alpha C)}\right]^{\prime}, \\
\mu \Phi=\left[12\left(1-\nu^{2}\right)\right]^{1 / 2} \frac{\alpha z^{\prime}}{r h}, \quad F=r^{\prime}\left(\frac{\alpha}{r D}\right)^{1 / 2}(r V), \\
G=r^{\prime}\left(\frac{\alpha C}{r}\right)^{1 / 2} k+\left(\frac{r}{\alpha C}\right)^{1 / 2}\left\{\left[\frac{r^{\prime} z^{\prime}}{r^{2}}+\nu \frac{\left(z^{\prime} / \alpha C\right)^{\prime}}{(r / \alpha C)}\right](r V)+\nu \frac{z^{\prime}}{r}(r V)^{\prime}\right. \\
\left.-\left[\frac{(r / \alpha C)^{\prime}}{(r / \alpha C)}+\nu \frac{r^{\prime}}{r}\right]\left(r \alpha p_{H}\right)-\left(r \alpha p_{H}\right)^{\prime}\right\},
\end{aligned}
$$

where $C=E h, D=E h^{3} / 12\left(1-\nu^{2}\right), E$ is Young's modulus, $\nu$ is Poisson's ratio and $h$ is the wall thickness of the shell. Note that the quantity $\mu \Phi$ is inversely proportional to the thickness $h$ and so is large compared to unity if $h$ is sufficiently small compared to other dimensions.

Quantities $p_{H}$ and $p_{V}$ denote radial and axial components of surface load intensity, while $V$ is an axial stress resultant determined by overall static equilibrium. Normal stress resultants $N_{\xi}$ and $N_{\theta}$, transverse shear resultant $Q$, and stress couples $M_{\xi}$ and 11 are all defined in the usual way. The components of displacement of a point of the middle shell surface in the three coordinate directions $r, \theta, z$ are denoted by $u, v, w$, respectively, while $\beta$ denotes the angle of rotation, due to deformation, of a tangent to a meridian. Although all other quantities are assumed to be independent of $\theta$, the circumferential component of displacement $v$ is allowed to have the form

$$
v=k r \theta \quad(k=\text { constant })
$$

which occurs when a sector of a shell is subjected to pure bending in a place perpendicular to the axis of revolution (see [8]). Once differential Eqs. (1) have been solved subject to appropriate boundary conditions, the various quantities may be determined from the following relations,

$$
\begin{aligned}
r V & =-\int r \alpha p_{V} d \xi \\
r N_{\xi} & =r^{\prime}\left(\frac{C}{r \alpha}\right)^{1 / 2} Y+\frac{z^{\prime}}{\alpha}(r V), \quad r Q=-z^{\prime}\left(\frac{C}{r \alpha}\right)^{1 / 2} Y+\frac{r^{\prime}}{\alpha}(r V), \\
\alpha N_{\theta} & =\left(\frac{\alpha C}{r}\right)^{1 / 2}\left[Y^{\prime}-\frac{1}{2} \frac{(r / \alpha C)^{\prime}}{(r / \alpha C)} Y\right]+r \alpha p_{H} \\
\alpha M_{\xi} & =\left(\frac{\alpha D}{r}\right)^{1 / 2}\left\{X^{\prime}+\left[\nu \frac{r^{\prime}}{r}-\frac{1}{2} \frac{(r D / \alpha)^{\prime}}{(r D / \alpha)}\right] X\right\} \\
\alpha M_{\theta} & =\left(\frac{\alpha D}{r}\right)^{1 / 2}\left\{\nu X^{\prime}+\left[\frac{r^{\prime}}{r}-\frac{\nu}{2} \frac{(r D / \alpha)^{\prime}}{(r D / \alpha)}\right] X\right\} \\
\beta & =\left(\frac{\alpha}{r D}\right)^{1 / 2} X, \quad u=\frac{r}{C}\left(N_{\theta}-\nu N_{\xi}\right) \\
w & =-\int\left[r^{\prime} \beta-\frac{z^{\prime}}{C}\left(N_{\xi}-\nu N_{\vartheta}\right)\right] d \xi .
\end{aligned}
$$


3. Asymptotic integration when $\Phi$ has a first-order zero. Approximate solutions of differential equations (1) can often be obtained by methods of asymptotic integration provided the parameter $\mu$ in these equations is sufficiently large compared to unity. The form and completeness of the results depends on the nature of the coefficient functions $\theta_{1}, \theta_{2}$, and $\Phi$. For instance, if these functions are all bounded and if $\Phi$ is also bounded from zero, asymptotic expansions in $\mu$ can be found which represent solutions of either the non-homogeneous differential equations or the corresponding homogeneous equations and which involve only elementary functions. Such expansions have been given for general shells of revolution by F. B. Hildebrand [5]. If $\Phi$ vanishes at some transition point or if $\theta_{1}$ and $\theta_{2}$ have at most second-order poles, as they usually do on the axis of revolution, asymptotic solutions can be obtained for the homogeneous differential equations using methods developed by $R$. E. Langer [6a, b] which involve Bessel functions. In general, Langer's methods yield only the leading terms of asymptotic expansions of solutions but may be used to find higher-order terms if the shell is such that Eqs. (1) can be reduced to a single second-order differential equation. Reduction of Eqs. (1) to a single second-order equation is possible whenever the shell thickness and the meridianal curvature are constant or, in general, whenever the ratio $\left(\theta_{1}-\theta_{2}\right) / \Phi$ is constant, as has been shown recently by P. M. Naghdi [7] who also lists the form of Langer's asymptotic solutions.

The present paper is concerned with asymptotic solutions of non-homogeneous equations (1) assuming that all ronfficient functions and loading terms ar. lannded functions of $\xi$ for the shell or portion of shell being considered and that $\Phi$ vanishes at some isolated point. According to Eqs. (4), $\Phi$ vanishes whenever $z^{\prime}(\xi)$ vanishes or whenever the tangent to a meridian is perpendicular to the axis of revolution. Define $\xi$ so that $\xi=0$ corresponds to the point where $\Phi$ is zero and let the range of $\xi$ be restricted, if necessary, so that $\Phi$ is bounded from zero away from $\xi=0$. Expanding about $\xi=0$ we have

$$
\mu \Phi(\xi)=\left[12\left(1-\nu^{2}\right)\right]^{1 / 2}\left\{\xi\left[\frac{\alpha z^{\prime \prime}}{r h}\right]_{\xi=0}+\xi^{2}\left[\frac{\alpha z^{\prime \prime}}{2 r h}+z^{\prime \prime}\left(\frac{\alpha}{r h}\right)^{\prime}\right]_{\xi=0}+\cdots\right\} .
$$

It follows that the coefficient of the first power of $\xi$ in expansion (7) is different from zero only if $z^{\prime \prime}(0) \neq 0$ or only if the radius of curvature of a meridian is finite at $\xi=0$. The function $\mu \Phi$ then is said to have a first-order zero and this is the only case we will consider. The parameter $\mu$ may be conveniently defined now as the value of the coefficient of $\xi$ in (7). Using a zero subscript to denote the value of a quantity at $\xi=0$ we may write

$$
\mu=\left[12\left(1-\nu^{2}\right)\right]^{1 / 2} \frac{\alpha_{0} z_{0}^{\prime \prime}}{r_{0} h_{0}}=\left[12\left(1-\nu^{2}\right)\right]^{1 / 2} \frac{\alpha_{0}^{3}}{r_{0} h_{0} R_{0}},
$$

where $R_{0}$ is the radius of curvature of a meridian at $\xi=0$. Dividing expansion (7) by formula (8) we now have

$$
\Phi(\xi)=\xi+A_{0} \xi^{2}+\cdots
$$

where

$$
A_{0}=\frac{1}{2} \frac{z_{0}^{\prime \prime \prime}}{z_{0}^{\prime \prime}}+\frac{\alpha_{0}^{\prime}}{\alpha_{0}}-\frac{r_{0}^{\prime}}{r_{0}}-\frac{h_{0}^{\prime}}{h_{0}}
$$


If there are no "rapid changes" in the shell surface or in its thickness, functions $\theta_{1}$ and $\theta_{2}$ are of order unity outside some neighborhood of the axis of revolution and so are small, generally, compared to $\mu \Phi$ provided $\mu$ is sufficiently large. This suggests that approximate solutions of differential equations (1) can be found by neglecting terms in $\theta_{1}$ and $\theta_{2}$. The resulting differential equations may be interpreted as the real and imaginary parts of a single complex differential equation of the form

$$
Z^{\prime \prime}-i \mu \Phi Z=f
$$

where

$$
Z=X+i Y, \quad f=F+i G .
$$

Thus, if Eqs. (1) cannot be reduced to a single second-order differential equation in an exact manner, they may be approximated by such an equation. The nature of the error in the approximation is discussed later on.

We consider now the problem of obtaining an approximate solution of differential equation (11) when $\mu$ is large. If we assume that a solution can be represented by an expansion in inverse powers of $\mu$, we obtain the following formal solution

$$
Z=\frac{i f}{\mu \Phi}+\frac{1}{\mu^{2} \Phi}\left(\frac{f}{\Phi}\right)^{\prime \prime}+\cdots
$$

which obviously is not valid in some neighborhond of $\xi=0$ where $\Phi$ vanishes. However, over an interval where $\Phi$ is bounded from zero a finite number of terms of (13) does furnish an approximate solution of (11). The first term of (13) will be called the membrane solution of (11), or of differential equations (1), since retaining only this term leads to the same results as the so-called membrane theory of shells. An approximate solution of Eq. (11) which is valid at $\xi=0$ and which reduces to the membrane solution away from $\xi=0$ may be constructed as follows. First, in the immediate neighborhood of $\xi=0$ differential equation (11) may be approximated by

$$
Z^{\prime \prime}-i \mu \xi Z=f(0),
$$

where we assume that $f(0) \neq 0$. A solution of (14) is given by $Z=\mu^{-2 / 3} f(0) T\left(\mu^{1 / 3} \xi\right)$ provided the function $T(x)$ is a solution of

$$
T^{\prime \prime}(x)-i x T(x)=1 .
$$

(Henceforth, primes are used to denote differentiation with respect to the written or implied argument of a function.) Let $T(x)$ stand for that (unique) solution of (15) which has the behavior $T(x) \sim i / x$ as $|x| \rightarrow \infty$ and consider the expression

$$
Z_{1}(\xi)=\mu^{-2 / 3} f(\xi) T\left(\mu^{1 / 3} \Phi\right) \text {. }
$$

Near $\xi=0, \Phi \sim \xi$ and $f(\xi) \sim f(0)$ so that $Z_{1}$ approximates a solution of (14) or (11). Away from $\xi=0, \mu^{1 / 3} \Phi$ is large for large $\mu$ so that $Z_{1} \sim i f / \mu \Phi$. Consequently, $Z_{1}$ furnishes an "interpolation" between a solution of Eq. (14) and the membrane solution of (11). The real and imaginary parts of the required function $T(x)$ and its derivative are tabulated briefly in [2]. Also the following representations hold, 


$$
\begin{aligned}
T(x) & =-\int_{0}^{\infty} \exp \left(-i x t-\frac{1}{3} t^{3}\right) d t \\
& =-\sum_{n=0}^{\infty}(-i)^{n} 3^{(n-2) / 3} \Gamma\left(\frac{n+1}{3}\right) \frac{x^{n}}{n !} \\
& \sim \sum_{n=0}^{\infty} \frac{i^{3 n+1}(3 n) !}{3^{n} n ! x^{3 n+1}}, \quad|x|>>1 .
\end{aligned}
$$

It can be shown that the real and imaginary parts of $Z_{1}$ are truly asymptotic solutions of differential equations ( 1 ) in the sense that the relative error approaches zero uniformly as $\mu \rightarrow \infty$. Even so, $Z_{1}$ may not yield sufficiently accurate results when $\mu$ is only moderately large making it desirable to have higher-order approximations to solutions of Eqs. (1) or (11). Recently [3] complete asymptotic expansions were found which represent a solution of a non-homogeneous second-order differential equation such as (11). They are of the form

$$
\begin{aligned}
& Z_{2}(\xi)= \mu^{-2 / 3} \sum_{n=0}^{\infty} \mu^{-n / 3} \sum_{k=0}^{[2 n / 3} a_{2 n, 2 n-3 k}(\xi) T^{(2 n-3 k)}(x), \\
& Z_{3}(\xi)=\mu^{-2 / 3} T(x) \sum_{n=0}^{\infty} \mu^{-n} a_{n}(\xi)+\mu^{-1} \sum_{n=0}^{\infty} \mu^{-n} b_{n}(\xi) \\
&+\mu^{-4 / 3} T^{\prime}(x) \sum_{n=0}^{\infty} \mu^{-n} c_{n}(\xi),
\end{aligned}
$$

where $T^{(k)}(x)$ denotes the $k$ th derivative of $T$ with respect to $x$ and

$$
x=\mu^{1 / 3}(3 \omega / 2)^{2 / 3}, \quad \omega=\int_{0}^{\xi} \Phi^{1 / 2}(\xi) d \xi .
$$

The first term of expansion (18) is similar to the expression for $Z_{1}$, while expansion (19) is analogous to expansions obtained by Langer [6c] for solutions of a homogeneous second-order differential equation. The various coefficients in either expansion may be successively determined so that the expansion formally satisfies differential equation (11). The expansions do not converge, in general, but a finite number of terms of either furnishes an approximate solution with an error of the order in $\mu$ of the first term neglected. Depending on the circumstances one expansion may be easier to use than the other but one may also interpret expansion (19) as simply a "rearrangement" of the terms in (18) accomplished by using differential equation (15) to express higher derivatives of $T$ in terms of $T$ and $T^{\prime}$. If Eq. (11) is only an approximation of Eq. (1), it would not be consistent to retain arbitrarily many terms in $Z_{2}$ or $Z_{3}$, considered now as approximate solutions of differential equation (11). In [3] it is shown that terms of relative order $\mu^{-2 / 3}$ in either $Z_{2}$ or $Z_{3}$ depend on terms such as those in $\theta_{1}$ and $\theta_{2}$ in differential equations (1). This fact leads to the result that when $\mu$ is large the relative error in approximating a solution of Eqs. (1) by a solution of Eq. (11) is of the order $\mu^{-2 / 3}$, making it generally inconsistent to retain more than the first two terms in the expansions for $Z_{2}$ and $Z_{3}$. But if Eqs. (1) can be reduced to a second-order equation in an exact manner, then theoretically asymptotic solutions with arbitrarily many terms can be found. 
For later reference we now list the explicit form of the first three terms in the two expansions for a solution of differential equation (11), namely

$$
\begin{gathered}
Z_{2}(\xi)=\frac{1}{\mu^{2 / 3}} f Q^{4} T(x)+\frac{i}{\mu} Q^{3}\left(Q^{3} f\right)^{\prime} T^{\prime \prime}(x) \\
+\frac{i}{\mu^{4 / 3}}\left\{Q^{7} Q^{\prime \prime} f T^{\prime}(x)-\frac{1}{2} Q^{3}\left[Q^{2}\left(Q^{3} f\right)^{\prime}\right]^{\prime} x T^{\prime \prime}(x)\right\}, \\
Z_{3}(\xi)=\frac{1}{\mu^{2 / 3}} f(0) Q T(x)+\frac{i}{\mu}\left[\frac{f}{\Phi}-\frac{f(0)}{Q^{3} \Phi}\right]+\frac{i}{\mu^{4 / 3}} \frac{f(0) T^{\prime}(x)}{2 Q \Phi^{1 / 2}} \int_{0}^{\xi} \frac{Q^{\prime \prime}}{Q \Phi^{1 / 2}} d \xi,
\end{gathered}
$$

where

$$
Q(\xi)=(3 \omega / 2)^{1 / 8} / \Phi^{1 / 4}, \quad Q(0)=1 .
$$

Note that definitions (23) and (20) imply $x=\mu^{1 / 3} \Phi Q^{4}$ and also that $d x / d \xi=\mu^{1 / 3} / Q^{2}$. If $T(x)$ is replaced by its asymptotic form $i / x$ when $x$ is large both (21) and (22) reduce to the membrane solution if $/ \mu \Phi$ plus terms of the same order as the second term in expansion (13). When $x=O(1)$ it follows that $\xi=O\left(\mu^{-1 / 3}\right)$. Then if $f, \Phi$, and $Q$ are expanded in powers of $\xi$ and terms of relative order $\mu^{-2 / 3}$ are neglected, expressions (21) and (22) both reduce to

$$
\begin{array}{r}
Z=\frac{f(0)}{\mu^{2 / 3}} T\left(\mu^{1 / 3} \xi\right)+\frac{1}{\mu}\left\{\frac{A_{0}}{5} f(0)\left[\mu^{2 / 3} \xi^{2} T^{\prime}\left(\mu^{1 / 3} \xi\right)-\mu^{1 / 3} \xi T\left(\mu^{1 / 3} \xi\right)\right]\right. \\
\left.+i\left[f^{\prime}(0)-\frac{3}{5} A_{0} f(0)\right]\right\},
\end{array}
$$

where $A_{0}$ is defined by Eq. (10). Although expression (16) for $Z_{1}$ also reduces to the membrane solution when $x$ is large, for $x=O(1)$ it reduces to an expression that agrees with only the first term of (24), showing that the relative error in $Z_{1}$ is of the order $\mu^{-1 / 3}$, in general.

The asymptotic solutions described above are applied to shell problems in the following sections.

4. Toroidal shells of arbitrary cross section. We shall consider two particular problems involving toroidal shells. One problem is the bending of a curved tube assuming that the tube forms a sector of a shell of revolution. The other is the deflection of a toroidal expansion joint due to an axial force, where the expansion joint consists of a toroidal shell slit around its inner circumference and joined at the edges to sections of

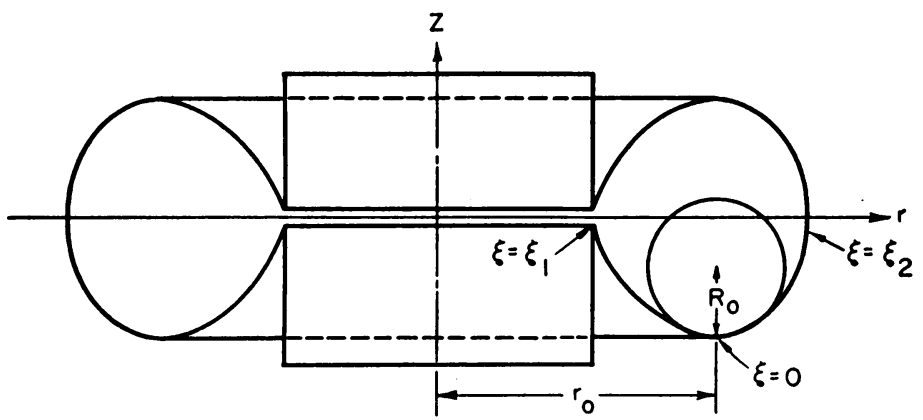

FIG. 1. Cross section of toroidal-shell expansion joint. 
cylindrical pipe (see Fig. 1). As will be seen, the two problems are mathematically quite similar. Asymptotic solutions of both have been given previously for toroidal shells of circular cross section and uniform thickness $[2,4]$. For the present we assume only that the shell has a plane of symmetry perpendicular to the axis of revolution.

If $P$ denotes the axial force applied to the expansion joint the loading terms in differential equations (1) become

$$
F=r^{\prime}\left(\frac{\alpha}{r D}\right)^{1 / 2} \frac{P}{2 \pi}, \quad G=\left[\frac{r^{\prime} z^{\prime}}{r^{2}}+\nu \frac{\left(z^{\prime} / \alpha C\right)^{\prime}}{(r / \alpha C)}\right]\left(\frac{r}{\alpha C}\right)^{1 / 2} \frac{P}{2 \pi} .
$$

Generally $G$ is small compared to $F$ and if coefficients $\theta_{1}$ and $\theta_{2}$ are neglected in Eqs. (1) it is consistent to neglect $G$ also. The right side of differential equation (11) is then given by the first of Eqs. (25).

For the problem of a tube or sector of a shell of revolution bent by end moments applied in a plane perpendicular to the axis of revolution we have

$$
F=0, \quad G=r^{\prime}\left(\frac{\alpha C}{r}\right)^{1 / 2} k .
$$

The constant $k$ is the same as that in Eq. (5) and is related to the change in curvature $\Delta K$ of the neutral surface of a tube by $k=r_{n} \Delta K$, where $r_{n}$ is the radius of the cylindrical neutral surface. For tubes with closed cross section the applied moment $m$ is given by

$$
m=\int\left(r-r_{n}\right) N_{\theta} \alpha d \xi .
$$

After differential equations (1) have been solved and stress resultants obtained in terms of $k$, the relationship between $k$ or $\Delta K$ and the applied moment $m$ may be established using (27).

Let the plane $z=0$ be the plane of symmetry for either problem and consider only the lower half of the shell, as shown in Fig. 1. Let $\xi_{1}$ and $\xi_{2}$ correspond to the inner and outer points, respectively, where a meridian meets the plane of symmetry. For the tube bending problem it follows from symmetry that $\beta=0$ and $Q=0$ at both $\xi_{1}$ and $\xi_{2}$. These conditions also hold at $\xi_{2}$ for the expansion joint but may not be the actual edge conditions at $\xi_{1}$. However, we shall assume that $\beta=0$ and $Q=0$ at $\xi_{1}$ for the expansion joint also, since it can be shown that axial deflection and maximum stresses are not significantly affected by a change in the conditions at $\xi_{1}$. Then, according to Eqs. (6) and (12), the boundary conditions for either problem are equivalent to

$$
Z\left(\xi_{1}\right)=0, \quad Z\left(\xi_{2}\right)=0 .
$$

The closed meridians of the toroidal shell are assumed to have continuously turning tangents which implies that $r^{\prime}$ vanishes at $\xi_{1}$ and $\xi_{2}$. Since the assumed loading term $f(\xi)$ for either problem involves $r^{\prime}$ as a factor, it follows that the membrane solution if $/ \mu \Phi$ vanishes at $\xi_{1}$ and $\xi_{2}$ so that asymptotic solutions $Z_{1}, Z_{2}$, and $Z_{3}$ all satisfy boundary conditions (28), at least to within terms of the order of the error involved. This means that no solution of the corresponding homogeneous differential equation is required and that approximate expressions for the various stress resultants and couples and displacements can be obtained from the real and imaginary parts of $Z_{1}, Z_{2}$, or $Z_{3}$ together with Eqs. (6). To evaluate the stresses and displacements as functions of $\xi$ one must first tabulate the functions $\Phi, Q$, and $x$ given by Eqs. (4), (20), and (23). These functions 
in turn depend on the exact shape of a meridian of the middle surface and on any variation in the shell thickness. Nevertheless, maximum stresses and other quantities of interest can be obtained explicitly without assuming any specific shape for the shell cross section.

Since $T(x) \sim i / x$ for large $x$ the most significant values of $Z$ as given by (16), (21), or (22) occur where $x=O(1)$ or $\xi=O\left(\mu^{-1 / 3}\right)$. For this range of $\xi$ expression (24) may be used as an approximate solution of differential equation (11) with a relative error of order $\mu^{-2 / 3}$. When the real and imaginary parts of (24) are substituted in Eqs. (6) it becomes apparent that for large values of $\mu$ the most significant stresses are the circumferential direct stress $\sigma_{\theta D}$ and the meridianal bending stress $\sigma_{\xi B}$, defined by

$$
\sigma_{\theta D}=\frac{N_{\theta}}{h}, \quad \sigma_{\xi B}=\frac{6 M_{\xi}}{h^{2}} .
$$

The maximum numerical values of these stresses and their location can be determined in a straightforward way using the following information about the real and imaginary parts of $T(x)$, denoted by $T_{r}(x)$ and $T_{i}(x)$. The maximum value of $T_{i}^{\prime}(x)$ occurs at $x=0$ where one has

$$
T_{r}(0)=-1.288, \quad T_{i}(0)=0, \quad T_{r}^{\prime}(0)=0, \quad T_{i}^{\prime}(0)=.939 .
$$

Tie maximum value of $T_{r}^{\prime}(x)$ occurs at $r=1.225$ where

$T_{r}(1.225)=-.692, \quad T_{i}(1.225)=.817, \quad T_{r}^{\prime}(1.225)=.753, \quad T_{i}^{\prime}(1.225)=.214$.

Also, $T_{r}(x)$ is an even function of $x, T_{i}(x)$ an odd function of $x$.

For the expansion joint problem we find after various calculations that

$$
\begin{gathered}
\sigma_{\theta D, \max }=\sigma_{\theta D}\left(\xi_{D}\right)=.342\left(1-\nu^{2}\right)^{1 / 3}\left(\frac{R_{0} h_{0}}{r_{0}^{2}}\right)^{1 / 3} \frac{P}{h_{0}^{2}}\left[1+O\left(\frac{1}{\mu^{2 / 3}}\right)\right] \\
\sigma_{\xi B, \max }=\sigma_{\xi B}\left(\xi_{B}\right)= \pm \frac{.475}{\left(1-\nu^{2}\right)^{1 / 6}}\left(\frac{R_{0} h_{0}}{r_{0}^{2}}\right)^{1 / 3} \frac{P}{h_{0}^{2}}\left[1 \pm \frac{2.14 A_{1}+.92 A_{2}}{\mu^{1 / 3}}+O\left(\frac{1}{\mu^{2 / 3}}\right)\right],
\end{gathered}
$$

where

$$
\begin{aligned}
& \xi_{D}=-\frac{.73}{\mu^{2 / 3}} \frac{r_{0}^{\prime}}{r_{0}}+O\left(\frac{1}{\mu}\right) \\
& \xi_{B}= \pm \frac{1.225}{\mu^{1 / 3}}+\frac{1}{\mu^{2 / 3}}\left(.30 A_{0}-.70 A_{2}\right)+O\left(\frac{1}{\mu}\right)
\end{aligned}
$$

with

$$
\begin{aligned}
& A_{1}=\frac{1}{10} \frac{z_{0}^{\prime \prime \prime}}{z_{0}^{\prime \prime}}-\frac{7}{10} \frac{r_{0}^{\prime}}{r_{0}}-\frac{3}{10} \frac{\alpha_{0}^{\prime}}{\alpha_{0}}-\frac{7}{10} \frac{h_{0}^{\prime}}{h_{0}}, \\
& A_{2}=(1-\nu) \frac{r_{0}^{\prime}}{r_{0}}+2 \frac{h_{0}^{\prime}}{h_{0}}
\end{aligned}
$$

As before a zero subscript denotes the value of a quantity at $\xi=0$. The plus and minus signs in the formula for $\sigma_{\xi B}$ correspond, respectively, to the signs in $\xi_{B}$ and the constant $A_{0}$ in $\xi_{B}$ is defined by Eq. (10). 
The total axial deflection or spread of the expansion joint due to bending only is given by

$$
\delta=2\left[w\left(\xi_{2}\right)-w\left(\xi_{1}\right)\right]=-2 \int_{\xi_{1}}^{\xi_{2}} r^{\prime} \beta d \xi .
$$

Since the integral extends over the full range of $\xi$, expression (24) is no longer appropriate. If the first two terms of $Z_{3}$ are used instead and $\beta$ is determined according to Eqs. (6), the expression for $\delta$ takes the form

$$
\delta=-\frac{r_{0}^{\prime} P}{\pi \mu}\left(\frac{\alpha_{0}}{r_{0} D_{0}}\right)^{1 / 2} \int_{x_{1}}^{x_{2}} r^{\prime}\left(\frac{\alpha}{r D}\right)^{1 / 2} Q^{3} T_{r}(x) d x,
$$

where $x_{1}$ and $x_{2}$ are values of $x$ corresponding to $\xi_{1}$ and $\xi_{2}$. Since $T_{r}(x) \sim 2 / x^{4}$ for large $x$, the major contribution of the integrand in (36) occurs for $x=0(1)$ or $\xi=0\left(\mu^{-1 / 3}\right)$. Also, interpreting $T(x)$ as a Fourier transform according to the integral representation given in (17) it follows that $\int_{0}^{\infty} T_{r}(x)=-\pi / 2$. Using these facts and introducing the explicit form of $\mu$ as defined in (8) it can be shown that

$$
\delta=\left[12\left(1-\nu^{2}\right)\right]^{1 / 2} \frac{R_{0} P}{E h_{0}^{2}}\left[1+O\left(\frac{1}{\mu^{2 / 3}}\right)\right] .
$$

This expression and the leading terms in expressions (32) are exactly the same as the corresponding results for a toroidal shell with a circular cross section of radius $R_{0}$ and a mean shell radius $r_{0}$, showing that when $\mu$ is sufficiently large the axial deflection and maximum stresses depend on the exact shape of the cross section only near the point where the slope $d z / d r$ of a meridian is zero.

For the tube bending problem results similar to (32) can be obtained with $E k$ in place of $P / h_{0}^{2}$. However, it is more convenient to obtain first the relationship between the applied moment $m$ and the constant $k$. Substituting for $\alpha N_{\theta}$ from Eqs. (6), with $Y$ taken as the imaginary part of $Z_{3}$ when $f=i k r^{\prime}(\alpha C / r)^{1 / 2}$, and integrating by parts, finally reduces formula (27) to

$$
m=-\frac{2 k r_{0}^{\prime}}{\mu}\left(\frac{\alpha_{0} C_{0}}{r_{0}}\right)^{1 / 2} \int_{x_{1}}^{x_{2}} r^{\prime}\left(\frac{\alpha C}{r}\right)^{1 / 2} Q^{3} T_{r}(x) d x,
$$

which is analogous to expression (36). Evaluating the integral in (38) in the same way as in (36) and introducing $\mu$ from Eq. (8), yields the result,

$$
m=\frac{2 \pi}{\left[12\left(1-\nu^{2}\right)\right]^{1 / 2}} R_{0} h_{0}^{2} E k\left[1+O\left(\frac{1}{\mu^{2 / 3}}\right)\right] .
$$

We recall that $k=r_{n} \Delta K$, where $\Delta K$ is the change in curvature of the neutral surface and $r_{n}$ is the radius of the neutral surface. Writing $r_{n}=r\left(\xi_{n}\right)$ the value of $\xi_{n}$ is by definition a solution of the equation $N_{\theta}-\nu N_{\xi}=0$. From expression (24) and Eqs. (6) one obtains

$$
\xi_{n}=-\frac{1.288}{\mu^{2 / 3}}\left[A_{1}+(1+\nu) \frac{r_{0}^{\prime}}{r_{0}}\right]+O\left(\frac{1}{\mu}\right),
$$

where $A_{1}$ is given by Eqs. (34). It follows from (40) that $r_{n}=r_{0}\left[1+0\left(\mu^{-2 / 3}\right)\right]$, so that expression (39) can also be written in the form

$$
m=\frac{2 \pi}{\left[12\left(1-\nu^{2}\right)\right]^{1 / 2}} r_{0} R_{0} h_{0}^{2} E \Delta K\left[1+O\left(\frac{1}{\mu^{2 / 3}}\right)\right] .
$$


This result may be compared with the equation $m=E I \Delta K$ which holds for a straight tube according to elementary beam theory, where $I$ is the moment of inertia of the cross section about an axis through the centroid. Writing

$$
m=\rho E I \Delta K
$$

for a curved tube serves to define the quantity $\rho$ as a rigidity factor which has the value unity for a straight tube. Comparing (41) and (42) it follows that

$$
\rho=\frac{2 \pi}{\left[12\left(1-\nu^{2}\right)\right]^{1 / 2}} \frac{r_{0} R_{0} h_{0}^{2}}{I}\left[1+O\left(\frac{1}{\mu^{2 / 3}}\right)\right]=\frac{2}{\mu} \frac{\pi \alpha_{0}^{3} h_{0}}{I}\left[1+O\left(\frac{1}{\mu^{2 / 3}}\right)\right] .
$$

Formula (43) is remarkably simple and shows that when $\mu$ is sufficiently large the effective stiffness $\rho E I$ of a curved tube depends on only the dimensions $r_{0}, R_{0}$ and $h_{0}$ associated with the point of the cross section where $\xi=0$. For a tube with circular cross section of radius $R_{0}$ and uniform thickness $h_{0}, I=\pi R_{0}^{3} h_{0}$. Also the quantity $\alpha$ may be identified with $R_{0}$ so that the rigidity factor then becomes simply $\rho=2 / \mu$ when $\mu$ is large.

The maximum stresses which occur in the bending of a curved tube may be compared with the maximum stress in a straight tube of circular cross section with radius $R_{0}$ and uniform thickness $h_{0}$ given by

$$
\sigma_{S M}=\frac{m R_{0}}{\mathrm{I}}=\frac{m}{\pi R_{0}^{2} h_{0}} .
$$

Introducing the expression for $m$ given in $n_{1}$. (39) and determining the maximum stresses in the same way as in the expansion joint problem we find

$$
\begin{aligned}
& \frac{\sigma_{\theta D, \max }}{\sigma_{S M}}=\frac{\sigma_{\theta D}\left(\xi_{D}\right)}{\sigma_{S M}}= \pm .862\left(1-\nu^{2}\right)^{1 / 3}\left(\frac{R_{0}^{2}}{r_{0} h_{0}}\right)^{2 / 3}\left[1 \pm \frac{2.14 A_{1}+.92\left(r_{0}^{\prime} / r_{0}\right)}{\mu^{1 / 3}}\right. \\
& \left.+O\left(\frac{1}{\mu^{2 / 3}}\right)\right] \\
& \frac{\sigma_{\xi B, \max }}{\sigma_{S M}}=\frac{\sigma_{\xi B}\left(\xi_{B}\right)}{\sigma_{S M}}=-\frac{1.86}{\left(1-\nu^{2}\right)^{1 / 6}}\left(\frac{R_{0}^{2}}{r_{0} h_{0}}\right)^{2 / 3}\left[1+O\left(\frac{1}{\mu^{2 / 3}}\right)\right],
\end{aligned}
$$

where now

$$
\begin{aligned}
& \xi_{D}= \pm \frac{1.225}{\mu^{1 / 3}}+\frac{1}{\mu^{2 / 3}}\left(.30 A_{0}-.70 \frac{r_{0}^{\prime}}{r_{0}}\right)+O\left(\frac{1}{\mu}\right) \\
& \xi_{B}=-\frac{.729}{\mu^{2 / 3}}+O\left(\frac{1}{\mu}\right) .
\end{aligned}
$$

The constant $A_{0}$ is defined by Eq. (10), $A_{1}$ and $A_{2}$ by Eqs. (34). The leading terms in (45) are the same as those for a tube with circular cross section of radius $R_{0}$. Thus, for sufficiently large $\mu$ the maximum stresses due to bending of a curved tube of arbitrary cross section can be obtained by considering an "equivalent" tube of circular cross section.

5. Toroidal shells of circular cross section. We now consider the form which the results in the previous section take for toroidal shells or curved tubes of circular cross section and uniform thickness $h$. We shall also list additional terms of relative order $\mu^{-2 / 3}$.

For shells of the special type now being considered parametric equations (2) may be taken as 


$$
r=a+b \sin \xi, \quad z=-b \cos \xi,
$$

so that $r_{0}=a, R_{0}=b, \alpha=b$. The parameter $\mu$ and a new parameter $\lambda$ are given by

$$
\lambda=\frac{b}{a}, \quad \mu=\left[12\left(1-\nu^{2}\right)\right]^{1 / 2} \frac{b^{2}}{a h} .
$$

Previous asymptotic solutions of the special problems being discussed did not take into account the effect of terms in $\lambda$ which was assumed to be negligibly small.

For the expansion-joint problem the largest values of the meridianal bending stress $\sigma_{\xi B}$ are now given by

$$
\dot{\sigma}_{\xi B}\left(\xi_{B}\right)= \pm .482\left(\frac{b h}{a^{2}}\right)^{1 / 3} \frac{P}{h^{2}}\left[1 \mp .86 \frac{\lambda}{\mu^{1 / 3}}+O\left(\frac{1}{\mu^{2 / 3}}\right)\right],
$$

where we have taken $\nu=.3$ and where

$$
\xi_{B}= \pm \frac{1.225}{\mu^{1 / 3}}-.79 \frac{\lambda}{\mu^{2 / 3}}+O\left(\frac{1}{\mu}\right) .
$$

It follows that the largest of the two values listed occurs at the "inner" point where $\xi_{B}$ is negative. For the tube-bending problem the only stress formula which involves $\lambda$ is the following for the maximum circumferential direct stress,

$$
\sigma_{\theta D}\left(\xi_{D}\right)= \pm .37 \tau_{\mu} \quad \sigma_{\sim M}\left[1 \mp .58 \frac{\lambda}{\mu^{1 / 3}}+O\left(\frac{1}{\mu^{2 / 3}}\right)\right],
$$

where again we have taken $\nu=.3$ and where

$$
\xi_{D}= \pm \frac{1.225}{\mu^{1 / 3}}-.997 \frac{\lambda}{\mu^{2 / 3}}+O\left(\frac{1}{\mu}\right) .
$$

From Eqs. (49) and (52) it is easily seen that terms in $\lambda$ play a minor role when $\lambda$ is small compared to $\mu^{1 / 3}$, which does not necessarily mean that $\lambda$ is small compared to unity as had been assumed in earlier work. However, the correction due to retaining terms in $\lambda$ may certainly be significant. For instance, when $\lambda=1 / 4$ and $\mu=8$ the correction to the maximum bending stress in the expansion joint, as given by Eq. (49), amounts to 10 per cent.

The relative size of the correction terms in $\lambda$ given above does not furnish any estimate of the accuracy of asymptotic formulas since at least part of the error term is independent of $\lambda$. Theoretically, exact expressions can be obtained for the terms of relative order $\mu^{-2 / 3}$ (or higher) but the necessary calculations become quite involved. However, if all terms in $\lambda$ are neglected higher-order terms in $\mu$ can be obtained without great effort. Neglecting all terms in $\lambda$ is equivalent to approximating differential equations (1) by the single complex equation

$$
Z^{\prime \prime}-i_{\mu} \sin \xi Z=f_{0} \cos \xi,
$$

where $f_{0}=\left(b^{3} / a D\right)^{1 / 2} P / 2 \pi$ for the expansion-joint problem and $f_{0}=i\left(b^{3} h E / a\right)^{1 / 2} k$ for the tube-bending problem. Neglecting terms in $\lambda$ is also equivalent to assuming that the various stresses and displacements are symmetrical with respect to $\xi=0$. An approximate asymptotic solution of (53) which includes terms of relative order $\mu^{-2 / 3}$ is given by either expression (21) or (22). To determine the most significant stresses one again 
restricts attention to the range where $\xi=0\left(\mu^{-1 / 3}\right)$ and expands all functions in powers of $\xi$. In this way the following results are finally obtained.

For the expansion-joint problem

$$
\begin{aligned}
\sigma_{\theta D, \max }=\sigma_{\theta D}(0) & =.342\left(1-\nu^{2}\right)^{1 / 3}\left(\frac{b h}{a^{2}}\right)^{1 / 3} \frac{P}{h^{2}}\left[1-\frac{.342}{\mu^{2 / 3}}+O\left(\frac{1}{\mu}\right)\right], \\
\sigma_{\xi B, \max }=\sigma_{\xi B}\left(\xi_{M}\right) & = \pm \frac{.475}{\left(1-\nu^{2}\right)^{1 / 6}}\left(\frac{b h}{a^{2}}\right)^{1 / 3} \frac{P}{h^{2}}\left[1-\frac{.054}{\mu^{2 / 3}}+O\left(\frac{1}{\mu}\right)\right], \\
\xi_{M} & = \pm\left[\frac{1.225}{\mu^{1 / 3}}-\frac{.017}{\mu}+O\left(\frac{1}{\mu^{4 / 3}}\right)\right], \\
\delta & =\left[12\left(1-\nu^{2}\right)\right]^{1 / 2} \frac{b P}{E h^{2}}\left[1+O\left(\frac{1}{\mu}\right)\right] .
\end{aligned}
$$

Analogously, for the tube-bending problem

$$
\begin{aligned}
& \sigma_{\theta D, \max }=\sigma_{\theta D}\left(\xi_{M}\right)= \pm .377 \mu^{2 / 3} \sigma_{S M}\left[1-\frac{.054}{\mu^{2 / 3}}+O\left(\frac{1}{\mu}\right)\right], \\
& \sigma_{\xi B, \max }=\sigma_{\xi B}(0)=-\frac{.814}{\left(1-\nu^{2}\right)^{1 / 2}} \mu^{2 / 3} \sigma_{S M}\left[1-\frac{.342}{\mu^{2 / 3}}+O\left(\frac{1}{\mu}\right)\right],
\end{aligned}
$$

where $\xi_{M}$ is again given by Eq. (55). Note that there is no "correction" term of order $\mu^{-2 / 3}$ in formula (56) for the axial deflection $\delta$ indicating that in an exact solution this term is probably proportional to $\lambda$. For the bending of a curved tube the analogous result for the rigidity factor is $\rho=(2 / \mu)[1+0(1 / \mu)]$.

In [4] where the leading terms in the above results for the tube-bending problem were first obtained, the asymptotic formulas are compared with numerical results calculated by L. Beskin [1] using a strain-energy principle in a manner equivalent to finding an exact trigonometric series solution of differential equation (53). In Tables 1 and 2 we have listed the numerical values obtained by retaining either one or two terms in formulas (57) and also the values obtained by Beskin.

TABLE 1.

Values of $\sigma_{\theta D, \max } / \sigma_{S M}$.

\begin{tabular}{|c|c|c|c|}
\hline$\mu$ & $.377 \mu^{2 / 3}$ & $.377 \mu^{2 / 3}-.020$ & Beskin [1] \\
\hline 1.00 & .377 & .357 & .979 \\
2.50 & .694 & .674 & .889 \\
3.54 & .875 & .855 & .881 \\
5.00 & 1.10 & 1.08 & 1.010 \\
7.07 & 1.39 & 1.37 & 1.277 \\
10.00 & 1.75 & 1.73 & 1.680 \\
12.50 & 2.03 & 2.01 & 1.99 \\
17.68 & 2.56 & 2.54 & 2.54 \\
25.00 & 3.22 & 3.20 & 3.26 \\
35.36 & 4.06 & 4.04 & 4.08 \\
50.00 & 5.11 & 5.09 & 5.09 \\
70.71 & 6.45 & 6.43 & 6.43 \\
100.00 & 8.13 & 8.11 & 8.12 \\
\hline
\end{tabular}


TABLE 2.

Values of $-\sqrt{1-\nu^{2}} \sigma_{\xi B, \max } / \sigma_{S M}$.

\begin{tabular}{|c|c|c|c|}
\hline$\mu$ & $.814 \mu^{2 / 3}$ & $.814 \mu^{2 / 3}-.278$ & Beskin $[1]$ \\
\hline 1.00 & .814 & .536 & .432 \\
2.50 & 1.50 & 1.22 & 1.06 \\
3.54 & 1.89 & 1.61 & 1.47 \\
5.00 & 2.38 & 2.10 & 2.01 \\
7.07 & 3.00 & 2.72 & .2 .69 \\
10.00 & 3.78 & 3.50 & 3.51 \\
12.50 & 4.38 & 4.10 & 4.11 \\
17.68 & 5.52 & 5.24 & 5.25 \\
25.00 & 6.96 & 6.68 & 6.67 \\
35.36 & 8.77 & 8.49 & 8.42 \\
50.00 & 11.0 & 10.7 & 10.7 \\
70.71 & 13.9 & 13.6 & 13.5 \\
100.00 & 17.5 & 17.2 & 17.2 \\
\hline
\end{tabular}

We observe that for $\sigma_{\theta D \text {, max }}$ the values found using the two-term asymptotic formula coincide with the values obtained by Beskin for $\mu \geq 50$ and differ from Beskin's results by less than 3 per cent for $\mu \geq 10$. Fnrmula (55) for the location of the maximum yields values which differ from those given by Beskin by less than 2 per cent for $\mu \geq 5$, and by less than 1 per cent for $\mu>10$, except for $\mu=50$ when there is a difference of more than 3 per cent.

From Table 2 for $\sigma_{\xi B, \max }$ we see that although the one-term asymptotic formula does not agree as closely with Beskin's results as does the one-term formula for $\sigma_{\theta D \text {, max }}$, the two-term formula yields values which differ from those found by Beskin by 1 per cent or less for $\mu \geq 7$, which is an even closer agreement than was found for $\sigma_{\theta D \text {, max }}$. In fact, the two-term asymptotic formula for $\sigma_{\xi B, \max }$ differs from Beskin's results by less than 5 per cent for $\mu$ as small as 5 .

Finally, it is of some interest to compare expressions (49) and (54). Unless $\lambda<.1 / \mu^{1 / 3}$ the correction to the one-term asymptotic solution due to retaining terms in $\lambda$ may be much more significant than that due to terms of relative order $\mu^{-2 / 3}$, at least as far as the dominant stress $\sigma_{\xi B}$ for the expansion-joint problem is concerned. A similar remark can be made about the circumferential direct stress $\sigma_{\theta D}$ for the tube-bending problem, although this stress is not the dominant one for this problem when $\mu$ is large. Also, the " $\lambda$-correction" to $\xi_{B}$ or $\xi_{D}$ in formulas (50) and (52) may be considerably more important than the second term in formula (55).

\section{REFERENCES}

1. L. Beskin, Bending of curved thin tubes, J. Appl. Mechanics, 12, A1-A7 1945)

2. R. A. Clark, On the theory of thin elastic toroidal shells, J. Math. and Phys. 29, 146-178 (1950)

3. R. A. Clark and W. E. Gibson, Asymptotic solution of a non-homogeneous differential equation, in preparation.

4. R. A. Clark and E. Reissner, Bending of curved tubes, Advances Appl. Mech. II, Academic Press, New York, 1951, 93-122

5. F. B. Hildebrand, On asymptotic integration in shell theory, Proc. Symposia Appl. Math. 3, McGrawHill, New York 1950, 53-66 
6. R. E. Langer, On the asymptotic solution of ordinary differential equations, Trans. Am. Math. Soc.(a) 33, 22-64 (1931); (b) 37, 397-416 (1935), (c) 67, 461-490 (1949)

7. P. M. Naghdi, On the deformation of elastic shells of revolution, Quart. Appl. Math. 12, 369-374 (1954)

8. E. Reissner, On bending of curved thin-walled tubes, Proc. Natl. Acad. Sci. U. S. 35, $204-208$ (1949)

9. E. Reissner, On the theory of thin elastic shells, Reissner Anniversary Volume, Edwards, Ann Arbor, 1949, 231-247 